NEIP-94-004

McGill-94/18

RAL/94-053

May 1994

\title{
ANOMALOUS VECTOR-BOSON COUPLINGS IN MAJORANA NEUTRINO MODELS
}

\author{
C.P. Burgess ${ }^{a}$ Fi $^{\dagger}$ and A. Pilaftsis ${ }^{b}$ \\ ${ }^{a}$ Institut de Physique, Université de Neuchâtel, 1 Rue A.L. Breguet, \\ CH-2000 Neuchâtel, Switzerland. \\ ${ }^{b}$ Rutherford Appleton Laboratory, Chilton, Didcot, Oxon, OX11 0QX, England.
}

\begin{abstract}
We examine the contributions of Majorana neutrinos to $C P$-violating $W W Z$ and $Z Z Z$ self-couplings, using a model in which sterile neutrinos couple to the $W$ and $Z$ by mixing with a fourth-generation heavy lepton. We find that the induced form factors can be as large as $0.5 \%$. The model satisfies all phenomenological bounds in a natural way, including those due to the strong limits on the neutron and electron electric dipole moments. Anomalous $C P$-odd couplings of this size are unlikely to be observed at LEP200, but might be detectable at NLC.
\end{abstract}

*Permanent address: Physics Department, McGill University, 3600 University St., Montréal, Québec, Canada, H3А $2 T 8$.

†E-mail addresses: cliff@physics.mcgill.ca, pilaftsis@vax2.rutherford.ac.uk 
The CERN Large Electron-Positron Collider (LEP), when operated at a centre-ofmass energy of $200 \mathrm{GeV}$ (LEP200), is expected to measure the three-point gauge-boson self-couplings, and so to either establish the non-Abelian structure predicted by the minimal Standard Model (SM), or to observe deviations from this that might signal the onset of new physics [1.2]. This prospect has stimulated many detailed theoretical examinations of the prospects for detecting these anomalous couplings at LEP200, or at a proposed $500 \mathrm{GeV}$ linear ee collider (NLC) [1,2, 3, 4, 4, 5,6,0,8,9,10]. All of the explicit models that have been proposed so far predict dauntingly small values - of order of $10^{-3}$ in appropriate units for the $C P$-odd $W W Z$ and $Z Z Z$ form factors. Since measurements are expected only to be sensitive to vector-boson self-couplings that are larger than or of order $10^{-2}$ (NLC) or $10^{-1}$ (LEP200), this suggests that any experimentally observed deviations from the SM cannot be understood within the framework of a perturbative, renormalizable field theory. We believe it to be worthwhile to explore this conclusion quantitatively, to see which scenarios maximize the expected anomalous form factors. We focus here on $C P$-violating couplings, since these are much easier to compute than are the $C P$-preserving ones. .

In this letter, we present a model which might be expected to produce anomalous $C P$-odd couplings through new physics that is naturally isolated from other observables, and so which is only quite weakly constrained by current data. The predicted anomalous couplings in this model therefore turn out to be comparatively large - up to $0.5 \%$ although only of a size to be detectable at the NLC. The model is based on supplementing the SM with a number of electroweak-singlet sterile neutrinos, which then couple to the electroweak bosons by mixing with a fourth-generation heavy neutrino which is a member of a conventional weak isodoublet. This kind of model has been previously considered as a potential contributor [12,13] to the oblique parameters [14] of precision electroweak measurements, [] as well as a model for producing $C P$-odd [16], and other quantum [17] effects in the Higgs sector.

\footnotetext{
*See, however, refs. [11].

${ }^{\dagger}$ Although the treatment of the oblique parameters in terms of the usual parameters $S, T$ and $U$ is not justified for neutrinos with masses near the electroweak scale [15], the bounds that were obtained in this way are not expected to qualitatively change in a complete treatment.
} 
We first briefly describe the model. We require, in addition to the usual SM particle content, a sequential heavy lepton, which we represent with a weak isodoublet, and isosinglet

$$
L_{L}^{0}=\left(\begin{array}{c}
N_{L}^{0} \\
E_{L}^{0}
\end{array}\right), \quad E_{R}^{0} .
$$

We imagine cancelling the electroweak anomalies of these fields by including also a fourth generation of quarks, although these cannot contribute to $C P$-violating anomalous gauge couplings at one loop, and so play no role in what follows. We finally add at least two right-handed sterile neutrinos, which we collectively denote as $N_{i R}^{0}, i=1, \ldots n \geq 2$. At least two species of sterile neutrinos are required in order to permit renormalizable $C P$-violating interactions amongst the neutrinos.

We assume for simplicity that the new sequential fourth-generation particles mix only very feebly with the first three generations, as is also required by global analyses of lowenergy data [20]. We do not suppress, however, any mixing amongst the sterile neutrinos, or between the sterile neutrinos and the fourth generation. The resulting left-handed mass matrix, M, for the heavy neutrinos then takes the following form [16]:

$$
\mathbf{M}=\left(\begin{array}{ll}
0 & \mu^{T} \\
\mu & M
\end{array}\right),
$$

where the first row and column correspond to the sequential fourth-generation neutrino, $\nu_{4} \equiv N_{0 L}^{0}$, which we label in what follows with the subscript ' 0 '. The rest of the rows and columns represent the various sterile neutrinos, $N_{i R}^{0}$. The quantities $\mu_{i}$ are generically complex numbers, but the matrix $M_{i j}=M_{i} \delta_{i j}$ can without loss be chosen to be diagonal, with real, nonnegative entries. The mass eigenstates and eigenvalues are obtained by diagonalizing $\mathbf{M}$ by a unitary matrix $\mathbf{U}$ as follows:

$$
\mathbf{U}^{T} \mathbf{M U}=\hat{\mathbf{M}}
$$

where the positive and diagonal matrix $\hat{\mathbf{M}}$ contains the mass eigenvalues of the heavy

\footnotetext{
${ }_{\ddagger}^{\ddagger}$ This type of model, but with only one sterile neutrino, has been studied with the goal of naturally accommodating a heavy fourth-generation neutrino [18], as well as for explaining the mass pattern of the light neutrinos 19 .
} 
neutrinos $N_{a}, a=0, \ldots, n$, along its diagonal. 3 The spectrum of exotic fermions also includes the heavy fourth-generation charged lepton, which we denote by $E$. As long as these new particles are heavier than $M_{Z} / 2$, so they are not produced in $e^{+} e^{-}$collisions at the $Z$ resonance, their masses and couplings are largely unconstrained.

In terms of these mass eigenstates, the charged- and neutral-current interaction of the heavy neutrinos become 21]

$$
\begin{aligned}
& \mathcal{L}_{\text {int }}^{W}=-\frac{g}{\sqrt{2}} W_{\mu} B_{E a} \bar{E} \gamma^{\mu} \mathrm{P}_{L} N_{a}+H . c ., \\
& \mathcal{L}_{\text {int }}^{Z}=-\frac{g}{4 c_{w}} Z_{\mu} \bar{N}_{a} \gamma^{\mu}\left[C_{a b} \mathrm{P}_{L}-C_{a b}^{*} \mathrm{P}_{R}\right] N_{b},
\end{aligned}
$$

where $\mathrm{P}_{L(R)}=\left(1-(+) \gamma_{5}\right) / 2, c_{w}$ is the cosine of the weak mixing angle, and the mixing matrices $B_{E a}$ and $C_{a b}$ are defined by

$$
B_{E a}=e^{i \delta_{E}} U_{0 a}^{*}, \quad C_{a b}=U_{0 a} U_{0 b}^{*}
$$

In Eq. (5), the phase $\delta_{E}$ is arbitrary and reflects the freedom to rephase the charged lepton field $E$. This phase can be used, for example, to ensure that $B_{E 1}$ is purely real. The remaining quantities, $B_{E i}$ for $i=2, \ldots, n$, are then generally complex, however, and their phases are the source of $C P$-violation which we shall use.

For the purposes of illustrating the possible neutrino spectrum and mixings, consider for a moment the case for which $\left|\mu_{i}\right| \ll M_{j}$, for all $i$ and $j$. In this case $n$ of the masseigenstate neutrinos are predominantly sterile, $N_{i}(i=1, \ldots, n)$, and have masses $M_{i}+$ $O\left(\mu^{2} / M\right)$. The remaining neutrino, $N_{0}$, is lighter, having mass $m_{0}$, where $m_{0}$ is the modulus of the following complex sum: $\sum_{i} \mu_{i}^{2} / M_{i} \equiv m_{0} e^{2 i \delta}$. The phase of this sum we call $2 \delta$. With this notation (and neglecting contributions of order $\mu^{2} / M^{2}$ ) the mixing angles are:

$$
U_{00}=i e^{i \delta}, \quad U_{0 i}=i e^{i \delta} \frac{\mu_{i}^{*}}{M_{i}}, \quad(i=1, \ldots, n)
$$

In what follows we do not wish to make the assumption that the $\mu_{i}$ are much smaller than the $M_{i}$. In this, the general case, it is more fruitful to work directly with the neutrino

\footnotetext{
${ }^{\S}$ We adopt here a notation for which indices from the middle of the alphabet, $i, j=1, \ldots, n$, label the predominantly sterile neutrinos, while indices from the beginning of the alphabet, $a, b=0, \ldots, n$ also include the dominantly isodoublet state, $N_{0}$.
} 
masses and mixings as our free parameters, keeping in mind that these are restricted by the following general identities [21,22]:

$$
C_{a b}=B_{E a}^{*} B_{E b}, \quad \sum_{c=0}^{n} m_{c} C_{a c} C_{b c}=0, \quad \sum_{c=0}^{n} m_{c} B_{E c} C_{c a}^{*}=0, \quad \sum_{c=0}^{n} m_{c} B_{E c}^{2}=0,
$$

where $m_{a}$ denotes the mass of the $n+1$ Majorana neutrinos.

In order to be completely concrete, we specialize at this point to the minimal case, for which we consider $n=2$ sterile neutrinos, and so for which we have three heavy neutrino mass eigenstates. In this case, as may be seen from Eqs. (6), the imaginary parts of $B_{E 1}$ and $B_{E 2}$ are related to one other via

$$
\operatorname{Im} B_{E 2}^{2}=-\frac{m_{1}}{m_{2}} \operatorname{Im} B_{E 1}^{2}
$$

where we have chosen the phase $\delta_{E}$ so that $\operatorname{Im} B_{E 0}=0$.

We now turn to the calculation of the $C P$-odd part of the transition element $W^{-\nu}\left(p_{1}\right) \rightarrow Z^{\mu}(q)+W^{-\kappa}\left(p_{2}\right)$ in this model. It is conventional to parametrize this in terms of the following form factors [1, 2]

$$
\left.\Gamma_{Z W W}^{\mu \nu \kappa}\right|_{C P-o d d}=-f_{Z}\left(q^{2}\right) \varepsilon^{\mu \nu \kappa \rho} q_{\rho}-\frac{g_{Z}\left(q^{2}\right)}{M_{W}^{2}} p^{\kappa} \varepsilon^{\mu \nu \sigma \rho} q_{\sigma} p_{\rho}+i h_{Z}\left(q^{2}\right)\left(q^{\mu} \mathrm{g}^{\nu \kappa}+q^{\nu} \mathrm{g}^{\mu \kappa}\right),
$$

where $p_{1}^{2}=p_{2}^{2}=M_{W}^{2}, p=p_{1}+p_{2}$, and $f_{Z}, g_{Z}, h_{Z}$ are $C P$-odd form factors. A similar analysis for the matrix element $Z^{\nu}\left(p_{1}\right) \rightarrow Z^{\mu}(q)+Z^{\kappa}\left(p_{2}\right)$ and assuming that the fields $Z^{\nu}$ and $Z^{\kappa}$ are on mass shell gives [1],2]

$$
\left.\Gamma_{Z Z Z}^{\mu \nu \kappa}\right|_{C P-o d d}=\frac{i \hat{h}_{Z}\left(q^{2}\right)}{M_{Z}^{2}}\left(q^{\mu} \mathrm{g}^{\nu \kappa}+q^{\nu} \mathrm{g}^{\mu \kappa}\right)
$$

where $\hat{h}_{Z}$ is the anapole form factor for the $Z Z Z$ vertex, and $\mathrm{g}_{\mu \nu}$ is the usual Minkowskispace metric.

For each of the form factors that appear in these expressions, there is a similar one in which $Z^{\mu}(q)$ is replaced with a photon. Of these, the two form factors, $f_{\gamma}$ and $g_{\gamma}$, are particularly dangerous, contributing as they do to the neutron and electron electric dipole moments (EDM's). As a consequence, these two are experimentally constrained to be rather small [4, 10]: quantitatively they must satisfy [23] $f_{\gamma}(0) \lesssim 10^{-3}$ and $g_{\gamma}(0) \lesssim 10^{-4}$. These bounds largely preclude the possibility of observing $f_{\gamma}$ and $g_{\gamma}$ in ee collisions for the forseeable future. 
Any viable model for producing a sizable $C P$-violating anomalous $W W Z$ or $Z Z Z$ interactions, must therefore not also produce the corresponding electromagnetic ones. One of the attractive features of sterile-neutrino models is that this is ensured in a completely natural way, because the $C P$-violating $W W \gamma$ and $Z Z \gamma$ couplings automatically vanish at one loop. For the $W W \gamma$ vertex, this vanishing arises because (in the absence of righthanded charged currents 惯河) any $C P$-violating phase in the $W$-fermion coupling cancels between the two $W$ vertices. Similar arguments hold for the $Z Z \gamma$ coupling. In this case the vanishing of the one-loop $C P$-odd $Z Z \gamma$ form factors is a consequence of the flavourdiagonal nature of the the $Z$ and $\gamma$ couplings to the charged leptons and quarks, as well as the absence of direct neutrino-photon couplings. Other contributions to light fermion EDM's are precluded by the assumed absence of mixing between the heavy and light leptons.

The same arguments do not rule out anomalous $W W Z$ and $Z Z Z$ couplings however. The difference is due to the possibility of having $C P$-violation and neutrino flavour changes at the $Z$-fermion vertices. At one loop only $f_{Z}$ and the anapole form factor, $h_{Z}$, turn out to be generated by Fig. 1(a) [10]. For the model at hand, we find

$$
f_{Z}\left(q^{2}\right)=-\frac{\alpha_{w}}{8 \pi c_{w}^{2}} \sum_{a b} \operatorname{Im} C_{a b}^{2} I\left(q^{2}, \lambda_{a}, \lambda_{b}, \lambda_{E}\right)
$$

where

$$
\begin{gathered}
I\left(q^{2}, \lambda_{a}, \lambda_{b}, \lambda_{E}\right)=\int_{0}^{1} \int_{0}^{1} d x d y y^{2}(1-2 x)\left[3 \ln \mathcal{A}^{W}\left(q^{2}, \lambda_{a}, \lambda_{b}, \lambda_{E}\right)\right. \\
\left.-\frac{q^{2}}{4 M_{W}^{2}}\left(\frac{1-y^{2}(1-2 x)^{2}}{\mathcal{A}^{W}\left(q^{2}, \lambda_{a}, \lambda_{b}, \lambda_{E}\right)}\right)\right], \\
\mathcal{A}^{W}\left(q^{2}, \lambda_{a}, \lambda_{b}, \lambda_{E}\right)=\lambda_{E}(1-y)+\lambda_{b} x y+\lambda_{a}(1-x) y-y(1-y) \\
-\frac{q^{2}}{M_{W}^{2}} y^{2} x(1-x)-i \varepsilon,
\end{gathered}
$$

and the kinematic variables $\lambda_{a}$ and $\lambda_{E}$ are defined as

$$
\lambda_{a}=\frac{m_{a}^{2}}{M_{W}^{2}}, \quad \lambda_{E}=\frac{m_{E}^{2}}{M_{W}^{2}}
$$

The summation over neutrino species in Eq. (10) may be simplified by using the identities of Eq. (6) to derive the following relations.

$$
\begin{aligned}
& \operatorname{Im} C_{02}^{2}=-\sqrt{\lambda_{1} / \lambda_{2}} \operatorname{Im} C_{01}^{2} \\
& \operatorname{Im} C_{12}^{2}=\sqrt{\lambda_{0} / \lambda_{2}} \operatorname{Im} C_{01}^{2} .
\end{aligned}
$$


These simplify Eq. (10) to:

$$
\begin{gathered}
f_{Z}\left(q^{2}\right)=-\frac{\alpha_{w}}{4 \pi c_{w}^{2}} \operatorname{Im} C_{01}^{2}\left[I\left(q^{2}, \lambda_{0}, \lambda_{1}, \lambda_{E}\right)-\sqrt{\lambda_{1} / \lambda_{2}} I\left(q^{2}, \lambda_{0}, \lambda_{2}, \lambda_{E}\right)\right. \\
\left.+\sqrt{\lambda_{0} / \lambda_{2}} I\left(q^{2}, \lambda_{1}, \lambda_{2}, \lambda_{E}\right)\right] .
\end{gathered}
$$

Using $\operatorname{Im} C_{01}^{2}=\mathcal{O}(1)$, in this expression gives the numerical estimates of Tables 1 and 2 for LEP200 and NLC, respectively. We find the largest values for $f_{Z}$ when the condition, $q^{2} \simeq\left(m_{0}+m_{1}\right)^{2}$, for threshold effects is satisfied, and these can be as large as $0.5 \%$. For heavy neutrinos, i.e. $m_{a} \gg M_{W}$, we find smaller values: $f_{Z} \lesssim 0.1 \%$. Unfortunately, LEP200 is likely to be unable to detect $C P$-violating anomalous $W$ - and $Z$-boson couplings that are smaller than $5-10 \%$ [8.9], and so these predictions are likely to be too small to be observed. Nevertheless, $C P$-odd form factors as small as $0.5-1 \%$ may be accessible at NLC, given an upgrade in the luminosity or the adoption of polarized $e^{+}$and $e^{-}$beams.

Our model also gives rise to an anapole form factor, $h_{Z}$, for the coupling $W W Z$ [10], again from the graph of Fig. 1(a). We find

$$
\begin{gathered}
h_{Z}\left(q^{2}\right)=\frac{\alpha_{w}}{4 \pi c_{w}^{2}} \operatorname{Im} C_{01}^{2}\left[K\left(q^{2}, \lambda_{0}, \lambda_{1}, \lambda_{E}\right)-\sqrt{\lambda_{1} / \lambda_{2}} K\left(q^{2}, \lambda_{0}, \lambda_{2}, \lambda_{E}\right)\right. \\
\left.+\sqrt{\lambda_{0} / \lambda_{2}} K\left(q^{2}, \lambda_{1}, \lambda_{2}, \lambda_{E}\right)\right],
\end{gathered}
$$

where

$$
\begin{array}{r}
K\left(q^{2}, \lambda_{a}, \lambda_{b}, \lambda_{E}\right)=\int_{0}^{1} \int_{0}^{1} d x d y y^{2}(1-2 x)\left[\ln \mathcal{A}^{W}\left(q^{2}, \lambda_{a}, \lambda_{b}, \lambda_{E}\right)\right. \\
\left.-\frac{q^{2}}{4 M_{W}^{2}}\left(\frac{1-y^{2}(1-2 x)^{2}}{\mathcal{A}^{W}\left(q^{2}, \lambda_{a}, \lambda_{b}, \lambda_{E}\right)}\right)\right] .
\end{array}
$$

Similarly, an anomalous anapole $Z Z Z$ coupling, $\hat{h}_{Z}$, is induced by the Feynman graph of Fig. 1(b) [10]:

$$
\begin{gathered}
\hat{h}_{z}\left(q^{2}\right)=-\frac{\alpha_{w}}{8 \pi c_{w}^{2}} \sum_{a b c}\left[\sqrt{\lambda_{a} \lambda_{b}} \operatorname{Im}\left(C_{a c} C_{a b} C_{b c}^{*}\right) L\left(q^{2}, \lambda_{a}, \lambda_{b}, \lambda_{c}\right)\right. \\
\left.+\operatorname{Im}\left(C_{a c}^{*} C_{a b} C_{b c}\right) \hat{K}\left(q^{2}, \lambda_{a}, \lambda_{b}, \lambda_{c}\right)\right],
\end{gathered}
$$

where

$$
L\left(q^{2}, \lambda_{a}, \lambda_{b}, \lambda_{c}\right)=\int_{0}^{1} \int_{0}^{1} d x d y \frac{y^{2}(1-2 x)}{\mathcal{A}^{z}\left(q^{2}, \lambda_{a}, \lambda_{b}, \lambda_{c}\right)},
$$




$$
\begin{gathered}
\hat{K}\left(q^{2}, \lambda_{a}, \lambda_{b}, \lambda_{c}\right)=\int_{0}^{1} \int_{0}^{1} d x d y y^{2}(1-2 x)\left[\ln \mathcal{A}^{Z}\left(q^{2}, \lambda_{a}, \lambda_{b}, \lambda_{c}\right)\right. \\
\left.-\frac{q^{2}}{4 M_{W}^{2}}\left(\frac{1-y^{2}(1-2 x)^{2}}{\mathcal{A}^{z}\left(q^{2}, \lambda_{a}, \lambda_{b}, \lambda_{c}\right)}\right)\right] \\
\mathcal{A}^{Z}\left(q^{2}, \lambda_{a}, \lambda_{b}, \lambda_{c}\right)=\lambda_{c}(1-y)+\lambda_{b} x y+\lambda_{a}(1-x) y-\lambda_{z} y(1-y) \\
-\frac{q^{2}}{M_{W}^{2}} y^{2} x(1-x)-i \varepsilon,
\end{gathered}
$$

and $\lambda_{z}=M_{Z}^{2} / M_{W}^{2}$. Eq. (18) can be significantly simplified by judiciously using Eq. (6). We find

$$
\begin{aligned}
& \operatorname{Im}\left(C_{a c} C_{a b} C_{b c}^{*}\right)=C_{c c} \operatorname{Im}\left(C_{a b}^{2}\right), \\
& \operatorname{Im}\left(C_{a c}^{*} C_{a b} C_{b c}\right)=0 .
\end{aligned}
$$

Taking Eqs. (14) and (22) into account, we arrive at our final expression

$$
\hat{h}_{Z}\left(q^{2}\right)=\frac{\alpha_{w}}{8 \pi c_{w}^{2}} \frac{\operatorname{Im} C_{01}^{2}}{\sqrt{\lambda_{2}}} \sum_{a b c d} \varepsilon_{a b c} C_{d d} \sqrt{\lambda_{a} \lambda_{b} \lambda_{c}} L\left(q^{2}, \lambda_{a}, \lambda_{b}, \lambda_{d}\right)
$$

where $\varepsilon_{a b c}$ is the usual Levi-Civita tensor. When using this expression to make numerical estimates, we assume that $C_{22} \ll 1$. In this case Eq. (24) simplifies to

$$
\hat{h}_{Z}\left(q^{2}\right) \simeq \frac{\alpha_{w}}{4 \pi c_{w}^{2}} \operatorname{Im} C_{01}^{2} \sqrt{\lambda_{0} \lambda_{1}}\left[L\left(q^{2}, \lambda_{0}, \lambda_{1}, \lambda_{0}\right)-L\left(q^{2}, \lambda_{0}, \lambda_{2}, \lambda_{0}\right)+L\left(q^{2}, \lambda_{1}, \lambda_{2}, \lambda_{0}\right)\right] .
$$

We present our numerical results for the anapole form factors, $h_{z}$ and $\hat{h}_{z}$, at the relevant collider energies $\left(\sqrt{q^{2}}=200 \mathrm{GeV}\right.$ and $\left.500 \mathrm{GeV}\right)$ in Tables 1 and 2. As may be seen from the tables, threshold effects can enhance the size of these couplings to the level of $\sim 0.5 \%$, which is on the edge of sensitivity at NLC.

Since the biggest contribution to the anomalous gauge couplings arises due to threshold-mass effects of the Majorana neutrinos $N_{0}, N_{1}$, and even these are at the edge of observability, one might expect to pair produce the intermediate neutrinos via reactions such as $e^{+} e^{-} \rightarrow N_{a} N_{b}$. Even if the heavy neutrinos should be sufficiently long-lived to escape the detector - such as if $m_{0} \lesssim m_{E}$, in which case $N_{0}$ cannot decay into charged leptons - then it is likely to be seen in measurements of the invisible $Z$ width at these energies. This can be probed by looking for events in which a hard photon, radiated from the initial electron/positron line, is seen to recoil against something invisible. The rate for 
producing a light neutrino pair, such as $N_{0} N_{0}$, normalized by the total SM invisible width is

$$
R_{\text {mis. }}=\frac{\sigma\left(e^{+} e^{-} \rightarrow N_{0} N_{0}\right)}{\sigma_{S M}\left(e^{+} e^{-} \rightarrow \text { invisible }\right)}=\left|C_{00}\right|^{2} \beta_{N_{0}} \frac{3+\beta_{N_{0}}^{2}}{12},
$$

where $\beta_{N_{0}}=\left(1-4 m_{N_{0}}^{2} / q^{2}\right)^{1 / 2}$ is the velocity of the outgoing $N_{0}$ in the centre of mass frame. For example, the rate for producing a $50 \mathrm{GeV}$ neutrino for $\sqrt{q^{2}}=200 \mathrm{GeV}$ would be $R_{m i s .} \simeq 25 \%$ if $C_{11} \simeq 1$. There is, however, a very narrow window of masses for which $q^{2}$ is just on the lower rise of the threshold enhancement, but for which there is insufficient energy for direct neutrino production.

As can also be seen from the tables, the couplings are larger for smaller values of the heavy charged-lepton mass (compare Table $1 \mathrm{a}$ with $1 \mathrm{~b}$, or $2 \mathrm{a}$ with $2 \mathrm{~b}$ ). If we restrict ourselves to the case where both the charged lepton $E$, and the Majorana neutrino $N_{0}$, are too heavy to be pair produced at the $q^{2}$ of interest, we are led to smaller results. For example, we find in this case $f_{Z} \lesssim 0.2 \%$, yielding $C P$-violating effects that are that much more difficult to detect.

In conclusion, we have demonstrated that Majorana-neutrino scenarios based on the SM gauge group can predict an anomalous $W W Z$ coupling $f_{Z} \lesssim 0.5 \%$. In principle, $C P$-violating effects due to the dispersive (absorptive) parts of anomalous couplings can be observed by looking at specific $C P T$-even (CPT-odd) observables in the decay products of $W$-boson pairs [3,8:9]. For example, effective $C P T$-even observables could be the forwardbackward asymmetry of the hardest jet when $W$ and $Z$ bosons decay hadronically or $P$-odd momentum correlations between the initial electrons and final charged leptons $[\mathbb{8}]$.

Acknowledgements. A.P. wishes to thank the University of Neuchâtel, and C.B. would like to thank the Institute for Theoretical Physics at Santa Barbara, for their kind hospitality. This research was partially funded by N.S.E.R.C. of Canada, les Fonds F.C.A.R. du Québec, the Swiss National Foundation, and the U.S. National Science Foundation (Grant No. PHY89-04035). 


\section{References}

[1] K.J.F. Gaemers and G.J. Gounaris, Z. Phys. C1 (1979) 259.

[2] K. Hagiwara, R.D. Peccei, D. Zeppenfeld, and K. Hikasa, Nucl. Phys. B282 (1987) 253.

[3] M.B. Gavela etal., Phys. Rev. D39 (1989) 1870; A. Bilal, E. Masssó, and A. de Rejula, Nucl. Phys. B355 (1991) 549; G.J. Gounaris, D. Schildknecht, and F.M. Renard, Phys. Lett. B263 (1991) 291.

[4] D. Atwood, C.P. Burgess, C. Hamzaoui, B. Irwin, and J.A. Robinson, Phys. Rev. D42 (1990) 3770.

[5] X.G. He and B.H.J. McKellar, Phys. Rev. D42 (1990) 3221; D. Chang et.al., Nucl. Phys. B355 (1991) 295.

[6] F. Boudjema, C.P. Burgess, C. Hamzaoui, and J. Robinson, Phys. Rev. D43 (1991) 3683.

[7] X.G. He, J.P. Ma, and B.H. McKellar, Phys. Lett. B304 (1993) 285.

[8] D. Chang, W.-Y. Keung, and I. Phillips, Phys. Rev. D48 (1993) 4045.

[9] M. Diehl and O. Nachtmann, Heidelberg preprint 1993, HD-THEP-93-37.

[10] C.P. Burgess, M. Frank, and C. Hamzaoui, McGill preprint McGill-93/40 hhepph/9312238).

[11] E.N. Argyres et.al., Nucl. Phys. B391 (1993) 23; M.J Herrero and E. Ruiz-Morales, Madrid preprint FTUAM-93-24, hep-ph/9308276 (unpublished).

[12] S. Bertolini and A. Sirlin, Phys. Lett. B257 (1991) 179; E. Gates and J. Terning, Phys. Rev. Lett. 67 (1991) 1840.

[13] B.A. Kniehl and H.-G. Kohrs, Phys. Rev. D48 (1993) 225. 
[14] M.E. Peskin and T. Takeuchi, Phys. Rev. Lett. 65 (1990) 964; Phys. Rev. D46 (1992) 381; G. Altarelli and R. Barbieri, Phys. Lett. B253 (1991) 161; G. Altarelli, R. Barbieri, and S. Jadach, Nucl. Phys. B369 (1992) 3.

[15] I. Maksymyk, C.P. Burgess, and D. London, McGill preprint McGill-93/13;

C.P. Burgess, H. König, D. London, and I. Maksymyk, Phys. Lett. B326 (1994) 276.

[16] A. Ilakovac, B.A. Kniehl, and A. Pilaftsis, Phys. Lett. B317 (1993) 609; (also Phys. Lett. B320 (1994) 329, published twice due to a publisher's error).

[17] B.A. Kniehl and A. Pilaftsis, DESY preprint 1994, DESY/94-020, Nucl. Phys. B (to appear).

[18] C.T. Hill and E.A. Paschos, Phys. Lett. B241 (1990) 96.

[19] K.S. Babu and E. Ma, Phys. Rev. Lett. 61 (1988) 674.

[20] P. Langacker and D. London, Phys. Rev. D38 (1988) 886; G. Bhattacharyya et al., Mod. Phys. Lett. A6 (1991) 2921; E. Nardi, E. Roulet, and D. Tommasini, Nucl. Phys. B386 (1992) 239; Phys. Rev. D46 (1992) 3040; C.P. Burgess, S. Godfrey, H. König, D. London, and I. Maksymyk, McGill preprint McGill-93/12 (hep-ph/9312291) Phys. Rev. D (in press).

[21] A. Pilaftsis, Z. Phys. C55 (1992) 275.

[22] A. Pilaftsis, Phys. Lett. B285 (1992) 68; J.G. Körner, A. Pilaftsis, and K. Schilcher, Phys. Rev. D47 (1993) 1080.

[23] W.J. Marciano and A. Queijeiro, Phys. Rev. D33 (1986) 3449; F. Boudjema, K. Hagiwara, C. Hamzaoui, and K. Numata, Phys. Rev. D43 (1991) 2223.

[24] Particle Data Group, K. Hikasa et al., Review of particle properties, Phys. Rev. D45 (1992) S1. 


\section{Figure and Table Captions}

Fig. 1: Feynman graphs responsible for generating anomalous $C P$-violating form factors in the vertices $W W Z$ and $Z Z Z$.

Tab. 1: Numerical estimates of the anomalous vector-boson couplings $f_{Z}, h_{Z}, \hat{h}_{Z}$ in units of $\operatorname{Im} C_{01}^{2}$ at $\sqrt{q^{2}}=200 \mathrm{GeV}$. We have used the values: (a) $m_{0}=m_{E}=$ $50 \mathrm{GeV}$ and $m_{2}=1 \mathrm{TeV}$, and (b) $m_{0}=m_{E}=100 \mathrm{GeV}$ and $m_{2} \gg 1 \mathrm{TeV}$.

Tab. 2: Numerical estimates of vector-boson $C P$-odd form factors in units of $\operatorname{Im} C_{01}^{2}$ at $\sqrt{q^{2}}=500 \mathrm{GeV}$. We have assumed the values: (a) $m_{0}=m_{E}=50 \mathrm{GeV}$ and $m_{2}=1 \mathrm{TeV}$, and (b) $m_{0}=m_{E}=300 \mathrm{GeV}$ and $m_{2} \gg 1 \mathrm{TeV}$. 
Table 1a

\begin{tabular}{|c|c|c|c|c|c|c|}
\hline $\begin{array}{r}m_{1} \\
{[\mathrm{GeV}]}\end{array}$ & $\operatorname{Re} f_{Z}$ & $\operatorname{Im} f_{Z}$ & $\operatorname{Re}_{Z}$ & $\operatorname{Im} h_{Z}$ & $\operatorname{Re} \hat{h}_{Z}$ & $\operatorname{Im} \hat{h}_{Z}$ \\
\hline 100 & $2.310^{-3}$ & $2.510^{-3}$ & $4.210^{-4}$ & $-1.610^{-3}$ & $-6.710^{-4}$ & $6.010^{-4}$ \\
\hline 150 & $5.310^{-3}$ & 0 & $-3.910^{-3}$ & 0 & $5.710^{-3}$ & 0 \\
\hline 200 & $2.110^{-3}$ & 0 & $-1.110^{-3}$ & 0 & $1.310^{-3}$ & 0 \\
\hline 300 & $1.510^{-3}$ & 0 & $-6.910^{-4}$ & 0 & $7.510^{-4}$ & 0 \\
\hline 400 & $1.210^{-3}$ & 0 & $-5.210^{-4}$ & 0 & $5.610^{-4}$ & 0 \\
\hline
\end{tabular}

Table 1b

\begin{tabular}{|r||r|r|r|}
\hline$m_{1}$ & $\operatorname{Re} f_{Z}$ & $\operatorname{Re}_{Z}$ & $\operatorname{Re} \hat{h}_{Z}$ \\
{$[\mathrm{GeV}]$} & & & \\
\hline \hline & & & \\
200 & $9.210^{-4}$ & $-3.810^{-4}$ & $3.710^{-4}$ \\
400 & $1.410^{-3}$ & $-5.110^{-4}$ & $4.910^{-4}$ \\
600 & $1.510^{-3}$ & $-5.410^{-4}$ & $4.810^{-4}$ \\
800 & $1.610^{-3}$ & $-5.610^{-4}$ & $4.710^{-4}$ \\
1000 & $1.710^{-3}$ & $-5.710^{-4}$ & $4.410^{-4}$ \\
& & & \\
\hline
\end{tabular}


Table 2a

\begin{tabular}{|c|c|c|c|c|c|c|}
\hline $\begin{array}{r}m_{1} \\
{[\mathrm{GeV}]}\end{array}$ & $\operatorname{Re} f_{Z}$ & $\operatorname{Im} f_{Z}$ & $\operatorname{Re}_{Z}$ & $\operatorname{Im} h_{Z}$ & $\operatorname{Re} \hat{h}_{Z}$ & $\operatorname{Im} \hat{h}_{Z}$ \\
\hline 100 & $-1.110^{-3}$ & $6.210^{-4}$ & $8.410^{-3}$ & $-3.310^{-4}$ & $-1.410^{-4}$ & $1.510^{-5}$ \\
\hline 200 & $-1.610^{-3}$ & $2.810^{-3}$ & $1.710^{-3}$ & $-1.710^{-3}$ & $-5.310^{-4}$ & $1.910^{-4}$ \\
\hline 300 & $3.610^{-4}$ & $5.210^{-3}$ & $7.010^{-4}$ & $-3.710^{-3}$ & $-7.310^{-4}$ & $8.310^{-4}$ \\
\hline 400 & $4.610^{-3}$ & $4.110^{-3}$ & $-3.010^{-3}$ & $-3.510^{-3}$ & $8.810^{-4}$ & $1.210^{-3}$ \\
\hline 450 & $5.510^{-3}$ & 0 & $-4.210^{-3}$ & 0 & $3.010^{-3}$ & 0 \\
\hline 500 & $2.910^{-3}$ & 0 & $-1.910^{-3}$ & 0 & $1.210^{-3}$ & 0 \\
\hline
\end{tabular}

Table 2b

\begin{tabular}{|r||r|r|r|}
\hline$m_{1}$ & $\operatorname{Re} f_{Z}$ & $\operatorname{Re}_{Z}$ & $\operatorname{Re} \hat{h}_{Z}$ \\
{$[\mathrm{GeV}]$} & & & \\
\hline \hline & & & \\
400 & $3.610^{-4}$ & $-1.510^{-4}$ & $1.410^{-4}$ \\
600 & $7.910^{-4}$ & $-3.010^{-4}$ & $2.910^{-4}$ \\
800 & $1.110^{-3}$ & $-3.810^{-4}$ & $3.710^{-4}$ \\
1000 & $1.210^{-3}$ & $-4.310^{-4}$ & $4.110^{-4}$ \\
1500 & $1.510^{-3}$ & $-5.110^{-4}$ & $4.510^{-4}$ \\
& & & \\
\hline
\end{tabular}


This figure "fig1-1.png" is available in "png" format from: http://arxiv.org/ps/hep-ph/9405349v1 